\title{
Perceived continuity of gliding and steady-state tones through interrupting noise
}

\author{
VALTER CIOCCA and ALBERT S. BREGMAN \\ McGill University, Montreal, Quebec, Canada
}

\begin{abstract}
Perceived continuity was studied by varying the direction (steady-state, upward glide, or downward glide), the frequency separation, and the slope of two sinusoidal tones separated by a louder burst of white noise. When the two tones had different directions, continuity was perceived according to a frequency-proximity principle (frequency-interpolation effect). On the other hand, when the tones had the same direction, continuity was perceived on the basis of a good-continuation principle (frequency-extrapolation, or frequency-trajectory, effect). We attempted to determine the degree of tuning of the frequency-extrapolation mechanism. Our results showed that there was some tuning for the starting frequency of the postnoise glide; in this case, the region of acceptance for continuity was centered around a frequency predicted from the trajectory of the prenoise glide. No evidence was found for tuning based on the slope of the sinusoidal tones. These results suggest that auditory processes need to analyze the postnoise sound before deciding whether the prenoise tone continued underneath the noise burst.
\end{abstract}

When portions of a sound are deleted and replaced by gaps of silence, the sound is perceived as interrupted. However, if the gaps of silence are replaced by a sound that is different and louder than the interrupted sound (e.g., if a tone is interrupted by a louder burst of noise), the fainter sound is heard as continuing underneath the louder one, even though it is not physically present at the same time as the louder sound. This phenomenon is known under the names of auditory continuity, auditory induction, pulsation threshold, and continuity effect, and it has been studied using speech and nonspeech sounds (Houtgast, 1972; Thurlow \& Elfner, 1959; Vicario, 1960; Warren, 1970; Warren \& Obusek, 1971). It has been shown that auditory continuity is likely to occur when a sound is alternated with or replaced by a potential masker (Houtgast, 1972; Warren, Obusek, \& Ackroff, 1972) or there is no evidence for boundaries belonging to the softer sound (Bregman \& Dannenbring, 1977; Warren et al., 1972). The study of the conditions of the stimuli in which continuity occurs allows us to more easily infer the laws that our perceptual system uses to organize complex acoustic input into meaningful perceptual units. We may consider continuity processes to be formed by heuristic rules (or principles of organization) that deal with the following problem: Are two sounds (e.g., two glided tones presented just before and after a louder burst of white noise) part of the same auditory object (giving, in this case, perceived continuity) or are they two distinct objects? According

Experiments 1 and 2 formed part of Valter Ciocca's master's thesis, submitted to McGill University in August 1985. Financial support came from the Natural Sciences and Engineering Research Council of Canada and from the Killam Research Fellowship program. We wish to thank Rhonda Amsel for advice on data analysis and Pierre Ahad for technical support. Reprints are available from A. S. Bregman, Psychology Department, McGill University, 1205 Docteur Penfield Ave., Montreal, PQ H3A 1B1, Canada. to the properties of these heuristic rules, which are determined by the structure and functioning of our nervous system, we will perceive two sounds separated by a louder noise as belonging to the same or to different auditory objects depending on the duration, intensity, and spectral characteristics of the stimuli.

The present study investigates the role of two cues for the perceptual integration of acoustic sequences, namely frequency proximity and alignment on a common trajectory. These are analogous to the Gestalt principles of grouping by proximity and good continuation (Wertheimer, 1912). The two cues were put in conflict by varying the frequency characteristics of the sounds preceding and following the louder noise burst.

The operation of a frequency-proximity principle should generate strong continuity effects when sounds preceding and following a louder burst of noise are close in frequency to each other. This can be seen in Figure 1, where the pattern is composed of an upward glide, a burst of white noise, and a steady-state tone. According to a frequency-proximity principle, Tone B in Figure 1 should give better continuity than Tone $A$.

On the other hand, if perceived continuity is determined by alignment on a common trajectory, stronger continuity should be obtained when the sounds preceding and following the burst of noise are aligned on the same trajectory on a log-frequency-by-time scale. The operation of a good-continuity principle should give best continuity with Tone A in Figure 1, which is placed at the exact frequency value that the glide would have reached if it had actually continued underneath the noise. This is called the trajectory frequency, or trajectory point, and is labeled $T$ in Figure 1. We have mentioned two cues for sequential integration, frequency proximity and alignment on a common trajectory. Going along with these, there are two ideas about the actual restoration process. The first is the 


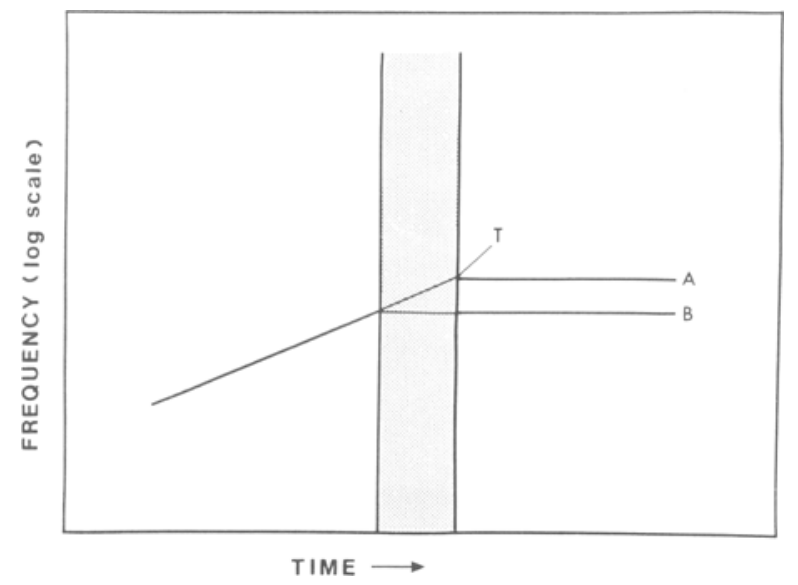

Figure 1. Tone $A$ is placed at the trajectory frequency of the prenoise glide, indicated by $T$; Tone $B$ matches the final frequency of the glide.

interpolation of a glide between the portions of the glide that are still present. This idea views restoration as analogous to stretching a rubber band between the last frequency estimate before the noise burst and the first one that follows it. The second idea about restoration sees it as the extrapolation of a glide along a trajectory that is partially missing. The mechanism might be viewed as placing a straight ruler along the existing portion of the glide and drawing a line (the restored glide) along the ruler.

The cue of alignment on a common trajectory seems to go most naturally with a restoration mechanism that involves extrapolation. The frequency-proximity cue for integration seems to go together most naturally with the interpolation (rubber band) method of restoration, since neither involves an extrapolation of frequency trajectories. Even so, one could imagine a case in which the decision about how strongly to consider the glides' entering and exiting from the noise as parts of the same sound was based on how well their trajectories lined up, but in which the pitch trajectory that was actually experienced by the listener was generated using the rubber-band method.

There has been an attempt to determine whether the auditory system can use the extrapolation method of restoration in the continuity of glided tones through noise (Dannenbring, 1976). In that experiment, connected sequences of alternately rising and falling glides (linear on a frequency $\times$ time scale) were presented. In one condition, the peaks (where the ascending glides turned into descending glides) were replaced by bursts of white noise. The listeners perceptually restored the turnaround points. Further investigation showed that the highest pitch the listeners actually heard was approximately the pitch that the ascending glides had reached before the noise (actually, it was even a bit lower). Therefore, the listeners did not extrapolate the pitch change into the noise, but sim- ply interpolated (stretched a rubber band) between the pitches on the two sides of the noise.

Although there was no extrapolation observed for the restoration process, one still might question whether alignment on a common trajectory might favor integration. In other conditions of Dannenbring's (1976) study, the glides were interrupted in the middle of rising and falling portions, but in these cases, since the remaining portions of the glides were always aligned on a common trajectory, it was not possible to answer this question.

In the present study, we tried to determine whether the process of perceptually integrating the glides that entered and exited from the noise could measure the trajectory of the entering glide and use that as a criterion for the good continuation of the exiting glide. We did not try to answer the question of whether the experience of restoration itself involved a rubber-band or a ruler-drawing mechanism. In fact, no direct assessment was made of the subjective pitch pattern in the perceptually restored sounds. Four experiments were conducted. In Experiment 1 , we employed acoustic patterns formed by a frequency glide, a burst of noise, and a steady-state tone in a sequence. The frequencies of the steady-state tones were varied to determine whether continuity was perceived on the basis of extrapolation or interpolation mechanisms. In Experiment 2, we examined whether perceived continuity depended on the relative direction of the sounds preceding and following the noise burst. Therefore, the direction (upward glide, downward glide, or steady-state) of the sine tones presented before and after the noise, as well as the initial frequency of the sound following the noise, were varied. In this experiment we found evidence for the existence of a trajectory effect when glides with the same direction were presented before and after the louder noise. In Experiments 3 and 4 we investigated the characteristics of the extrapolating mechanism by varying the starting frequency (Experiment 3 ) and the slope (Experiment 4) of the glide following the noise burst.

\section{EXPERIMENT 1}

The purpose of the present experiment was to discover the cues continuity-generating processes were based on when a logarithmic glide was followed by a louder burst of noise that was followed by a stationary tone.

The following factors were varied: the slope of the glide that preceded the noise (steep vs. shallow), the direction of the glides (upward vs. downward), and the frequency of the steady-state tone that followed the noise.

Let us consider, for simplicity, the predictions that we can make when the prenoise sounds are upward glides that have the same final frequency. If a trajectory-based criterion is operating, the frequencies of the postnoise sounds that give the best continuity effects should be significantly higher than the final frequencies of the glides. On the other hand, if continuity is based on a frequencyproximity criterion, it should be strongest when the post- 
noise sounds match the final frequencies of the glides, which are identical for shallow and steep prenoise glides.

\section{Method}

Subjects. Twenty-five undergraduate and graduate volunteers in psychology and nursing were tested. Of these, 5 subjects were discarded because they did not reach the criterion of 9 out of 10 correct answers in the pretest session. Therefore, 20 subjects participated in the experimental sessions. All of the subjects reported having no major hearing problems.

Stimuli. Three factors were considered in the experimental design: the steepness of the glide (Factor A), defined as a fullfrequency range ( 392 to $1396 \mathrm{~Hz}$, or 22 semitones in $.5 \mathrm{sec}$ ) or a half-frequency range (740 to $1396 \mathrm{~Hz}$ for upward glides and 740 to $392 \mathrm{~Hz}$ for downward glides, or 11 semitones in $.5 \mathrm{sec}$ ); the direction of the glide (upward or downward, Factor B); and the frequency of the steady-state tone (Factor C). The steady-state tones had 7 frequency values, defined as follows (see Figure 2): (1) the initial frequency of the glide; (2) the frequency at $1 / 2$ of the transition; (3) the frequency at $*$ of the transition; (4) the final frequency of the glide; (5) the half-trajectory frequency (or point), chosen arbitrarily to be in the middle of the frequency range delimited by the final frequency of the glide at one extreme and the trajectory frequency at the other extreme; (6) the trajectory frequency; (7) a frequency 3 semitones above (for ascending glides) or below (for descending glides) the trajectory frequency. The half-trajectory frequency was chosen, for the steeper glided tones, to match the trajectory frequency of the shallow glide. For the shallow glides, the halftrajectory frequency could not match any of the frequency values defined for the steeper glides; therefore, the semitone value closest to the actual center of the frequency range contained between the trajectory frequency and the final frequency of the glide was chosen. The steady-state tones and the glides used in the experiment were sinusoidal. The glides (shown in Figure 3 ) rose in frequency on a logarithmic scale from 392 to $1396 \mathrm{~Hz}$ (Glide A) and from 740 to $1396 \mathrm{~Hz}$ (Glide B), or fell in frequency from 1396 to $392 \mathrm{~Hz}$ (Glide C) and from 740 to $392 \mathrm{~Hz}$ (Glide D). The actual frequency values of the steady-state tones were: $392,740,1046,1396,1661$, 2093, and $2637 \mathrm{~Hz}$ for Glide A; 740, 1046, 1174, 1396, 1479, 1661, and $2093 \mathrm{~Hz}$ for Glide B; 1396, 740, 523, 392, 329, 261, and $207 \mathrm{~Hz}$ for Glide $\mathrm{C}$; and 740, 554, 466, 392, 369, 329, and $261 \mathrm{~Hz}$ for Glide D.

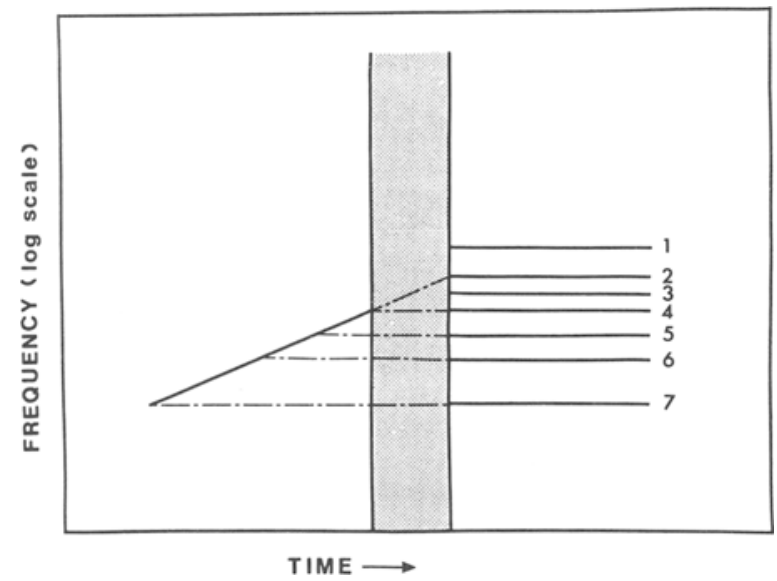

Figure 2. The seven steady-state tones used in Experiment 1. The tones are labeled as follows: (1) above trajectory frequency; (2) trajectory frequency; (3) $1 / 2$ trajectory frequency; (4) final frequency of the prenoise glide; (5) $*$ transition frequency; (6) $1 / 2$ transition frequency; (7) initial frequency of the glide.

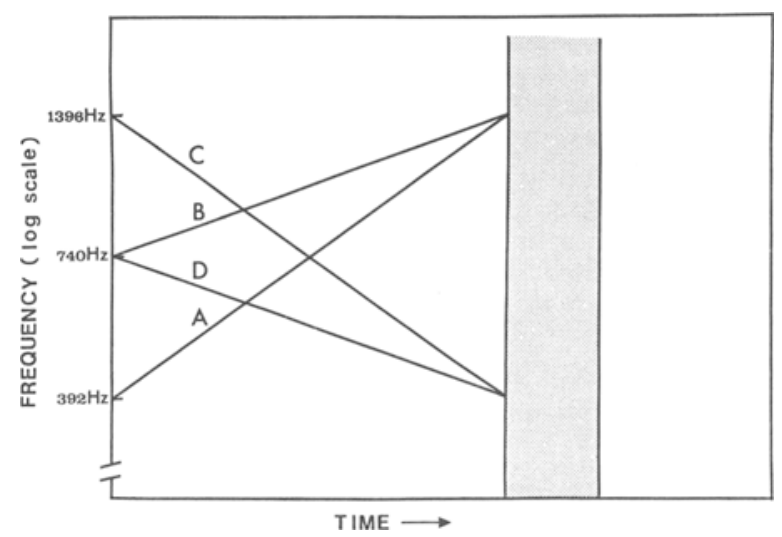

Figure 3. Frequency characteristics of the glides employed in Experiment 1.

All the steady-state and glided tones had a duration of $500 \mathrm{msec}$ and a level of $60 \mathrm{dBA}$. The onset time was $30 \mathrm{msec}$, the offset time $20 \mathrm{msec}$. The attack and decay envelopes were a quarter of a sine wave. The noise was a band of white noise, low-pass-filtered at $5000 \mathrm{~Hz}, 80 \mathrm{dBA}$ in intensity, and of a fixed duration of $150 \mathrm{msec}$. The transition between the tones and the noise was abrupt. Each experimental stimulus was repeated cyclically five times within each trial. There was $1 \mathrm{sec}$ of silence between the warning tone and the first presentation of a stimulus and between each stimulus in a trial. A 5-sec period separated each trial from the following trial. All 28 conditions were presented in random order within a session. There were two sessions of $9 \mathrm{~min}$ each, with a pause of about $5 \mathrm{~min}$ between the sessions.

Pretest. Ten stimuli were presented to the subjects in the pretest. Five stimuli had a sound that physically continued through the noise and the remaining stimuli were identical to the previous ones but had no sound present at the same time as the noise. The spectral and temporal properties of these stimuli were representative but did not match exactly those of the experimental stimuli. The bursts of noise were $5 \mathrm{dBA}$ louder than the sinusoidal tones (70 dBA). The subjects were told, at first, which stimuli had the sound present and which did not. A drawing showing presence and absence was also given. After this training, the subjects had to give a "present" or "absent" response to the same stimuli, presented in a different order. If the subjects gave less than 9 correct answers out of 10 trials, they had another chance to try to reach criterion. If they failed again, they were discarded.

Procedure. The subjects were tested individually in an audiometric room. They were told that their task was to detect the presence of a sound at the same time as the noise. During the main experiment the sound was never physically present in the experimental condition, although subjects were not told this. A 9-point rating scale was used: 1 was labeled "very sure Present" and 9 was labeled "very sure Not Present"; 5 was labeled "not sure." The listeners were encouraged to use, as much as possible, all the values on the scale and to use the score of $\mathbf{5}$ only if they could not decide at all whether the sound was present or absent. Subjects were also informed that the percentage of stimuli in which the sound was present was randomly determined by the computer, so that they could not expect any definite proportion of present stimuli. None of the subjects doubted that in some of the experimental stimuli a sound was really present at the same time as the noise.

Apparatus. The stimuli were digitally synthesized on a Digital Equipment Corporation PDP 11/34 computer, using the MITSYN software package (Henke, 1975), and played directly by the computer during the experiment. The stimuli were low-pass-filtered at 
$5000 \mathrm{~Hz}$ by a Rockland Dual Filter Model 852 and binaurally presented over Telephonics TDH-49P headphones in an Industrial Acoustics 1202 audiometer chamber. The intensities of the experimental stimuli at different frequencies were equalized using an ADC Sound Shaper Two graphic equalizer. The intensity levels of the stimuli were measured using a General Radio Type 1551-C sound-level meter set at " $A$ " weighting and connected to the headphones by a flat-plate coupler. The subjects used a keyboard to record their ratings.

\section{Results}

The data were analyzed using a three-way, repeated measures ANOVA, with the frequency factor nested within the other two factors; the mean of the ratings for each condition, obtained from each subject in the two sessions, was taken as the subject's score in that condition. The main effect of steepness was very reliable $[F(1,19)$ $=52.18, p<.0001]$ : steeper glides gave worse overall perceived continuity than did shallow ones. The main effect of the frequency of the steady-state tones was also highly significant $[F(24,456)=14.71, p<.0001]$. The means for each condition are displayed in the plots of Figure 4. A clear quadratic trend can be observed in all of the plotted curves, meaning that one or two frequency values were chosen as the best matches for continuity (indirectly measured as presence of sounds through noise) for each of the four glides preceding the noise, with other frequency values giving weaker continuity as they deviated from the best values. The lowest score values (best continuity) were given to stimuli in which the frequency of the steady-state tone was equal to or close to the final frequency of the glides. The steep-glide curves look quite similar in shape to the shallow-glide curves; however, the shallow glides give better overall continuity than do steep glides. Also, the shallow-slope curves are more sharply

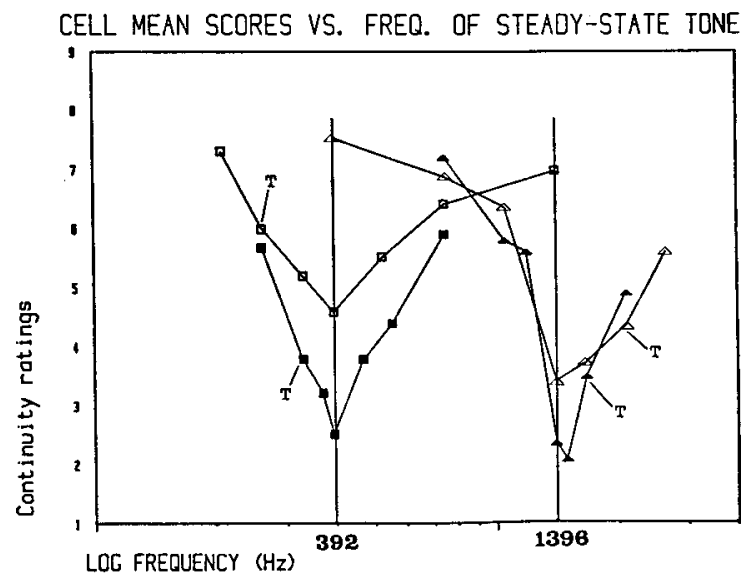

Figure 4. Mean continuity ratings for each of the four glides preceding the burst of white noise. High scores indicate weak continuity; low scores indicate strong continuity. The vertical lines indicate the final frequency of the downward glide (line on the left) and upward glide (line on the right). The trajectory-frequency condition for each glide is marked by a $T$. $\square$ : downward steep glide; $\square$ : downward shallow glide; $\triangle$ : upward steep glide; $\Delta$ : upward shallow glide. tuned than the steep-slope curves, which means that the latter accept a broader range of frequency matches. Finally, the curves for upward glides show a sharp continuity cutoff for frequency matches below the final frequency of the glide (there is a better acceptance for frequency matches above the final frequency of the glide than for frequencies below), and the curves for downward glides are more symmetrical around the final frequency value.

Finally, the interaction of steepness with direction was significant at the .01 level $[F(1,19)=9.16]$ : among steep glides, the upward glide gave significantly better continuity than the downward one and, among shallow glides, the downward glide gave stronger continuity than the upward one.

\section{Discussion}

The results of this experiment strongly support the idea that continuity is a process that operates on the basis of a frequency-proximity principle. That is, the closer the frequency of the steady-state tone is to the final frequency of the glide, the stronger are the obtained continuity effects. The area of acceptance for frequency matches seems to be influenced by the steepness of the glide: the steeper glides have a broader frequency range of acceptance for continuity than do the shallower ones. Moreover, frequency matches for continuity with upward glides are equal to or higher than the final frequency of the glides. On the other hand, with downward glides the frequency area of acceptance does not seem to be limited to frequencies at or below the final frequency of the glides. This difference could be due to the fact that the postnoise tones were sampled from the low frequency range for downward glides and from the high frequency range for upward glides.

Finally, both the main effect of steepness and the interaction of steepness with direction can be explained to be due to the worse continuity effects obtained with steeper glides, particularly with the steep downward glide. Similar results, showing that rising shallow-frequency glides were easier to follow and were perceived more as unitary events than as falling steep-frequency glides, were reported by Brady, House, and Stevens (1961).

In conclusion, no evidence has been found in this experiment, either from the statistical analysis of the data or from the pattern of cell means, that trajectory extrapolation is an important factor in the perceived continuity between a glided and a steady-state tone.

\section{EXPERIMENT 2}

The findings of Experiment 1 supported the hypothesis that perceived continuity is accomplished by finalfrequency interpolation, according to the principle of frequency proximity. The present experiment was done to determine whether the results of the previous experiment could be replicated using any other set of pre- and postnoise stimuli (e.g., glide/steady-state, glide/glide, or 


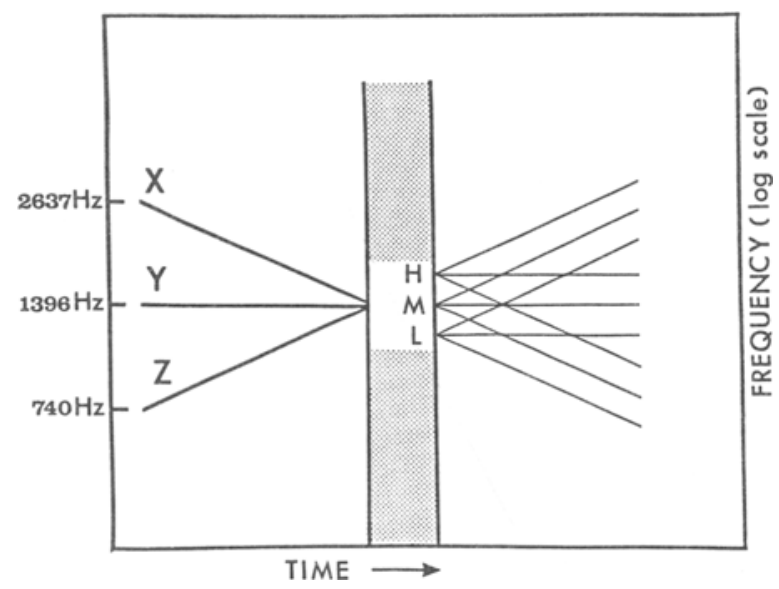

Figure 5. Experimental conditions in Experiment 2. The levels of the type of prenoise sound (sounds $X, Y$, and $Z$ ) are crossed with the levels of the starting frequency of the postnoise sound (H: high frequency; M: matching frequency; L: low frequency) and the type of the postnoise sound (upward glide, steady-state, or downward glide).

steady-state/glide), or whether they were due to the particular set of stimuli used in that experiment.

To look at the latter possibility, three factors were varied in Experiment 2: the type of the prenoise sounddownward glide, steady-state, or upward glide (see Figure 5, Sounds X, Y, and Z, respectively); the initial frequency of the postnoise sound, with three levels defined relative to the final frequency of the prenoise sound-the matching frequency, the matching frequency +3 semitones (high frequency), and the matching frequency -3 semitones (low frequency); and the type of the postnoise sound-upward glide, steady-state, or downward glide.

\section{Method}

Subjects. Twenty-five university students were employed, 8 of whom had participated in Experiment 1. Five subjects were discarded because they did not reach the criterion in the pretest session. One of the discarded subjects had reached criterion and had participated in the previous experiment. Twenty subjects participated in the experimental sessions. All of the subjects reported having no major hearing problems. All of the subjects were paid for their services.

Stimuli. The prenoise sounds (see Figure 5) had the following characteristics: the downward glide (Sound $\mathrm{X}$ ) moved in frequency from 2637 to $1396 \mathrm{~Hz}$, the steady-state tone (Sound Y) had a frequency of $1396 \mathrm{~Hz}$, and the upward glide (Sound Z) moved from 740 to $1396 \mathrm{~Hz}$. The slope of all the glides used in the experiment was kept constant in log-frequency-by-time coordinates, with a frequency difference of 11 semitones from the initial to the final frequency value ( 22 semitones per sec). This slope is the same as that of the shallow glides used in Experiment 1; moreover, the upward glide (Sound $\mathrm{Z}$ ) is identical to the shallow upward glide employed in that experiment. The initial frequency of the postnoise sound had three values: $1396 \mathrm{~Hz}$ (matching frequency), $1661 \mathrm{~Hz}$ (high frequency), this being the trajectory frequency in log-frequency-by-time coordinates for the first upward glide, and $1174 \mathrm{~Hz}$ (low frequency), this being the trajectory frequency for the prenoise downward glide. The actual frequency value for the trajectory frequency (in log- frequency-by-time coordinates) was 3 semitones and 30 cents (100 cents $=1$ semitone) above or below the final frequency of the glide. However, for the purpose of this experiment, it was more practical to choose an exact semitone value for the levels of the second factor. We believe that a difference of 30 cents from the actual trajectory-frequency value is not large enough to invalidate our inferences regarding the existence of trajectory effects in perceived continuity. The postnoise sound was either a steady-state tone, an upward glide, or a downward glide. The actual frequency values of the glides were: for the high $(1661 \mathrm{~Hz})$ starting frequency, 1661 to $3135 \mathrm{~Hz}$ (upward glide) and 1661 to $880 \mathrm{~Hz}$ (downward glide); for the matching (1396 Hz) starting frequency, 1396 to $2637 \mathrm{~Hz}$ (upward glide) and 1396 to $740 \mathrm{~Hz}$ (downward glide); and for the low $(1174 \mathrm{~Hz}$ ) starting frequency, 1174 to $2217 \mathrm{~Hz}$ (upward glide) and 1174 to $622 \mathrm{~Hz}$ (downward glide). All of the stimuli were identical to the stimuli of the previous experiment for duration, intensity, and spectral characteristics. All 27 conditions were presented in random order within each session. There were two sessions of about $9 \mathrm{~min}$ each and a pause of about $5 \mathrm{~min}$ between the sessions.

Pretest. The pretest procedure was identical to the one used in Experiment 1 . Twenty stimuli were employed to familiarize the subjects with all of the stimulus patterns presented in the experimental sessions. Ten of the training stimuli ( 5 present and 5 absent) were presented in a first block of trials and the other 10 stimuli were presented in a second block (if this was necessary).

Procedure and Apparatus. The procedure and apparatus were the same as the ones used in the previous experiment.

\section{Results}

A three-way ANOVA with repeated measures was used to analyze the data; the mean of the ratings obtained from a subject in the two sessions for each condition was taken as the subject's score in that condition. Figure 6 presents the cell means for each experimental condition, separated into three plots according to the type of prenoise sound presented. The main effect for the initial frequency of the postnoise sound showed that sounds starting at the matching frequency gave better overall continuity than did sounds with high or low initial frequencies $[F(2,38)=$ $48.15, p<.0001]$. The type of postnoise-sound main effect was highly significant $[F(2,38)=39.56, p<.0001]$ : a stationary tone following the noise gave overall worse continuity than upward or downward glides. The main effect for the type of prenoise sound was not significant.

When the prenoise sound was a steady-state tone, the best continuity effects were obtained with postnoise sounds starting at the matching frequency. On the other hand, if the prenoise sound was an upward or downward glide, continuity was weak for initial frequencies within the frequency range of this sound and equally strong for matching and trajectory (high or low) frequencies. This effect (the interaction of type of prenoise sound with the starting frequency of the postnoise sound) was highly significant $[F(4,76)=25.86, p<.0001]$.

Dannenbring (1976) found strong continuity when alternating descending and ascending (mirror-image) glides were separated by a louder burst of noise. In the present experiment we replicated Dannenbring's results. For example, if an upward glide was present before the noise, the postnoise sound that gave the best continuity was a downward glide. On the other hand, if frequency values 


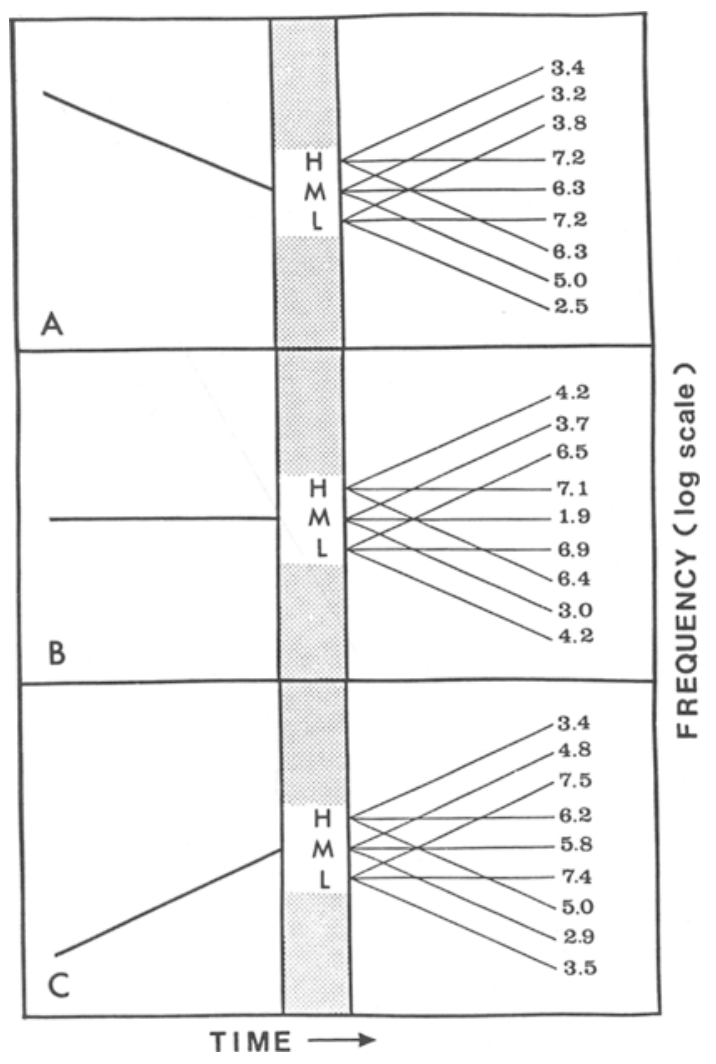

Figure 6. The mean continuity scores for each condition in Experiment 2 are displayed to the right in panels $A, B$, and $C$. Each panel illustrates the scores for the conditions associated with one of the three prenoise sounds.

just before and after the noise burst did not match (high or low initial frequencies), the best continuity was given by a sound whose direction was consistent with the direction of the frequency change between the end of the prenoise and the beginning of the postnoise sounds (goodcontinuity principle). The interaction of starting frequency with type of postnoise sound was significant at the .0001 level $[F(4,76)=36.64]$. The interaction of type of prenoise sound with type of postnoise sound was also highly significant $[F(4,76)=17.53, p<.0001]$. This effect means that if the prenoise sound was an upward glide, overall best continuity occurred when a downward glide followed the noise; symmetrical results were obtained when an upward glide preceded the noise.

Finally, the interaction between type of prenoise sound, starting frequency, and type of postnoise sound was significant at the .0001 level $[F(8,152)=4.74]$. This effect could be interpreted by saying that better continuity was obtained with different types of postnoise sounds for different combinations of prenoise sounds and initial frequencies (see Figure 6). A planned comparison was performed to see whether trajectory glides gave significantly better continuity than all other (nontrajectory) conditions for each of the two first glides. This effect was found to be significant at the .01 level.

\section{Discussion}

The results of this experiment can be summarized by saying that when pre- and postnoise sounds were of the same type (downward glides, upward glides, or steadystate tones), the best continuity was obtained with postnoise sounds starting at the trajectory frequency rather than at the matching frequency. On the other hand, when the sounds preceding and following the noise were of different types, a better continuity was obtained when the initial frequency of the postnoise sound matched the final frequency of the prenoise sound. This last finding agrees with the results of Experiment 1, showing that final-frequency interpolation is taking place with such patterns.

This interpretation of the results is in agreement with the idea that continuity is determined in a retrograde fashion (Vicario, 1973), because the auditory system does not know whether the first tone continued underneath the noise until it analyzes the characteristics of the second sound (that is, until some time after the noise burst).

In conclusion, the data of this experiment argue that the auditory system is capable of measuring both the frequency proximity and the alignment on a common trajectory of the sounds preceding and following the noise. Both measurements serve as criteria for integration. Further evidence is required in order to better specify the way the extrapolation mechanism functions, by systematically varying the starting frequency and the slope of the postnoise sounds.

\section{EXPERIMENT 3}

The purpose of Experiment 3 was to determine whether the trajectory-based integration mechanism was sharply tuned for the starting frequency of the second sound. If this were the case, continuity should be strongest when the postnoise sound started at the exact trajectory frequency of the prenoise sound. Two factors were varied in this experiment: the slope of the prenoise glide and the starting frequency of the postnoise glide. The slopes of the postnoise glides were kept constant and matched those of the glides preceding the noise. In Experiments 3 and 4 , we used only descending glides since the results obtained in Experiment 2 for ascending and descending glides were symmetrical.

\section{Method}

Subjects. Twenty-seven volunteers, all of whom were university students, were tested. Seven subjects were discarded because they failed to reach criterion in the pretest; thus, 20 subjects participated in the experimental sessions, 7 of whom had participated in Experiments 1 and/or 2. All subjects reported having no major hearing problems.

Stimuli. The prenoise sound was either a steep glide moving from 2637 to $1396 \mathrm{~Hz}$ (22 semitones per sec slope) or a shallower glide moving from 1975 to $1396 \mathrm{~Hz}$ (12 semitones per sec slope). The steeper glide had the same slope as the shallow glides from Experiment 1 and was identical to the first downward glide from Experiment 2 (Sound $X$ in Figure 5). For each of the two prenoise sounds, we presented six postnoise glides varying in starting fre- 
quency, but not in slope. The six starting frequencies were determined as proportions of the reference interval, which was the difference, measured in semitones, between the final frequency of the prenoise glide $(1396 \mathrm{~Hz})$ and the trajectory frequency of each of the two prenoise sounds (see Figure 7). More precisely, the starting frequencies were chosen by adding $1 / 2,0,-1 / 2,-1,-3 / 2$, and -3 of the reference interval to the final frequency of the prenoise sound. The reference interval for the steep glide, whose trajectory frequency was $1083 \mathrm{~Hz}$, was 4 semitones and 40 cents. The reference interval for the shallow glide was 2 semitones and 40 cents (1216 Hz trajectory frequency). In this experiment, as well as in Experiment 4, the actual trajectory frequencies were employed rather than approximations. The starting frequencies of the postnoise glides for the steep prenoise glide were: 1586 ( $1 / 2$ difference), 1396 (0 difference), 1230 ( $-1 / 2$ difference), 1083 ( -1 difference, or "trajectory frequency"), $954 \mathrm{~Hz}$ ( $-3 / 2$ difference), and $652 \mathrm{~Hz}$ (-3 difference). The final frequencies of the postnoise glides for the same sound were: $840,740,652,574,505$, and $345 \mathrm{~Hz}$, respectively. The initial frequencies of the postnoise glides for the shallow prenoise glide were: 1479 ( $1 / 2$ difference), 1396 (0 difference), 1303 ( $-1 / 2$ difference), 1216 ( -1 difference, or trajectory condition), 1135 ( $-3 / 2$ difference), and $922 \mathrm{~Hz}(-3$ difference). The final frequencies of these glides were $1059,988,922,860,802$, and $652 \mathrm{~Hz}$, respectively. The burst of white noise had the same spectral characteristics as the ones used in the first two experiments, but was 200 msec long. The reason for employing a longer noise duration was that in a pilot study we found that there was a tendency to perceive strong continuity with glides moving in the same direction. A longer noise duration was intended to give us more precise distinctions between the experimental conditions, because the reference interval would be larger (a glide under the noise would have moved farther in frequency). The stimuli were identical in intensity and spectral properties to those of Experiments 1 and 2. All conditions of the experiments were presented four times in random order in a 40-trial experimental session that lasted $15 \mathrm{~min}$. The same subjects were tested in Experiments 3 and 4, in two successive sessions. There was a 5-min break between the two sessions. The order of the experiments was balanced among subjects, so that one session was presented first to half of the subjects and the other session was presented first to the other half of the subjects.

Pretest. The training and test procedures were identical to those used in Experiment 1. The subjects received the training procedure before each of the two sessions; the screening procedure occurred only before the first session.

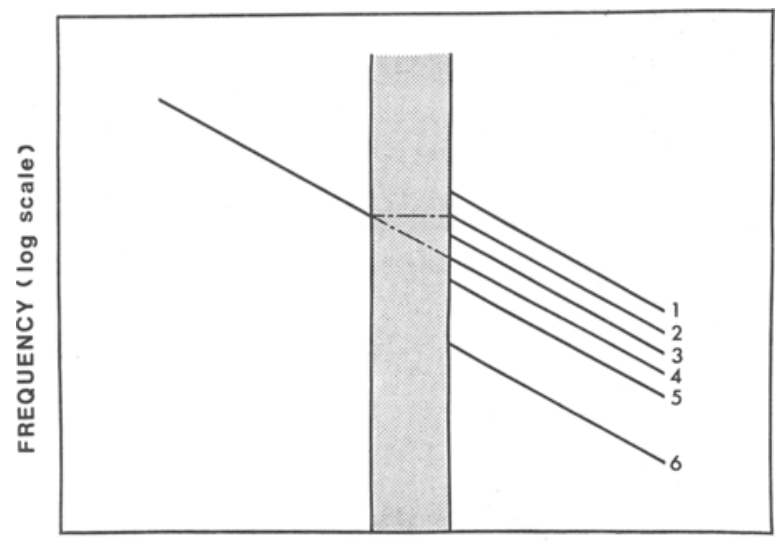

TIME $\longrightarrow$

Figure 7. Display of the levels of the starting frequency of the second sound in Experiment 3. The levels are shown as follows: (1) $1 / 2$ difference; (2) 0 difference; (3) $-1 / 2$ difference; (4) -1 difference; (5) $-3 / 2$ difference; (6) -3 difference.

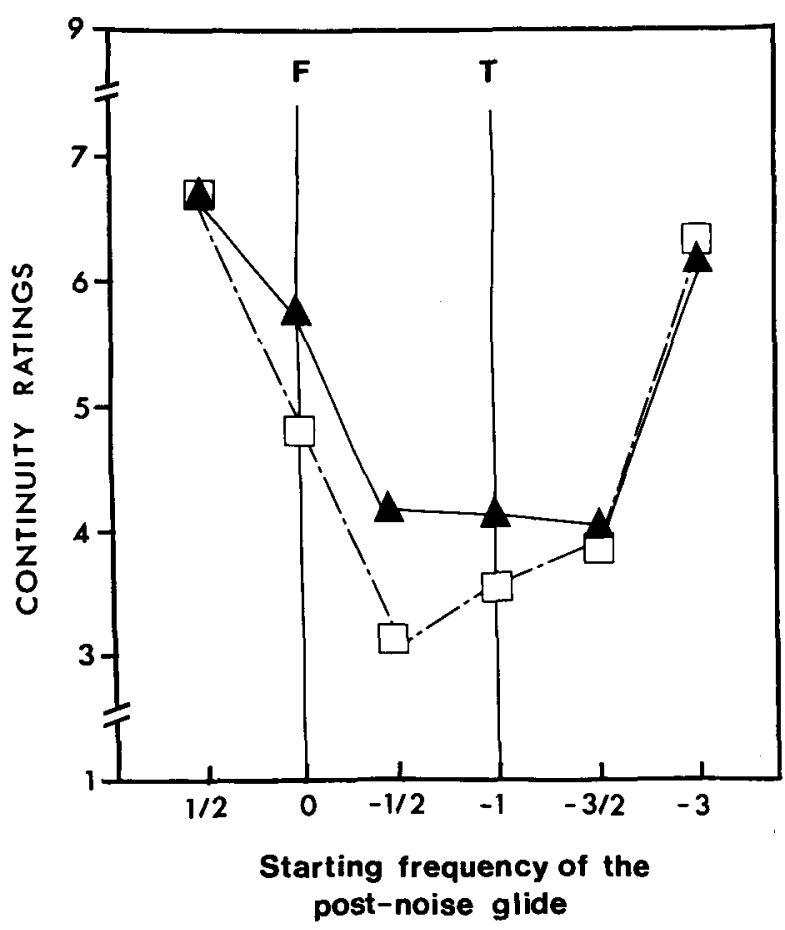

Figure 8. Mean continuity ratings in Experiment 3. Low scores indicate strong continuity. $\Delta$ : steep prenoise glide; $\square$ : shallow prenoise glide. The vertical lines indicate the final (F) and trajectory (T) frequencies of the prenoise glides.

Procedure and Apparatus. The procedure and apparatus were the same as those employed in Experiment 1.

\section{Results}

The results were analyzed in a two-way ANOVA with the factor of initial frequency of the postnoise glide nested within the factor of steepness of prenoise glide. Mean continuity ratings are shown in Figure 8 . The main effect of the initial frequency of the postnoise sound was highly significant $[F(10,190)=13.84, p<.0001]$. The main effect of prenoise glide steepness was not significant. Two planned comparison procedures were applied to test the size of the difference between conditions of $-1 / 2,-1$, and $-3 / 2$ differences and the other conditions, for each of the prenoise glides. These comparisons were found to be highly significant $(p<.0001)$. No pairwise post hoc comparison between conditions of $-1 / 2,-1$, and $-3 / 2$ differences was significant.

\section{Discussion}

The continuity ratings obtained in this experiment suggest the existence of a region of acceptance for continuity, situated in a frequency region around the trajectory frequency. This region of acceptance seems to be relatively broad and is centered at the trajectory frequency. This is in agreement with the results of Experiment 2. Continuity seems to be equally strong for all the postnoise glides that start within the region of acceptance. The pat- 
terns of cell means for the steep and shallow prenoise glides look very similar, as can be seen in Figure 8 . In agreement with the results of Experiment 1, the shallow glide seems to have a smaller frequency difference between the points of best and worst continuity, and therefore is more sharply tuned than the steeper glide.

The stimuli used in Experiment 3 are analogous to the visual stimuli that cause an underestimation of perceived visual trajectory (Poggendorff illusion; see, e.g., Weintraub \& Krantz, 1971). However, the results obtained with our stimuli show no evidence for a similar illusion in the auditory field.

\section{EXPERIMENT 4}

With Experiment 4, we wanted to see whether the trajectory-based mechanism would give improved continuity for postnoise glides that matched the prenoise glide in slope. In other words, we measured how perceived continuity changed when the slope of the sound following the noise was varied while keeping its starting frequency fixed at the trajectory frequency of the prenoise glide.

Two factors were varied in the experimental design: the slope of the prenoise glide and the slope of the postnoise glide.

\section{Method}

Subjects. The subjects were the same as those who participated in Experiment 3.

Stimuli. The prenoise sounds were identical to those employed in Experiment 3. The slope of the postnoise-glide factor had five levels, which were determined as proportions of the slope of the prenoise glide. The glides following the noise had a slope that was 0 (steady-state tone), 1/2, 1 (same slope, or trajectory glide), $3 / 2$, and 2 times the slope of the prenoise glide (see Figure 9). Therefore, for the steep prenoise glide the slopes of the postnoise glides were $0,11,22,33$, and 44 semitones per sec, respectively. The postnoise glides for the shallow prenoise glide had slopes of 0,6 , 12,18 , and 24 semitones per sec, respectively. The actual frequency values of the postnoise sounds were: 1086,1086 to 790,1086 to

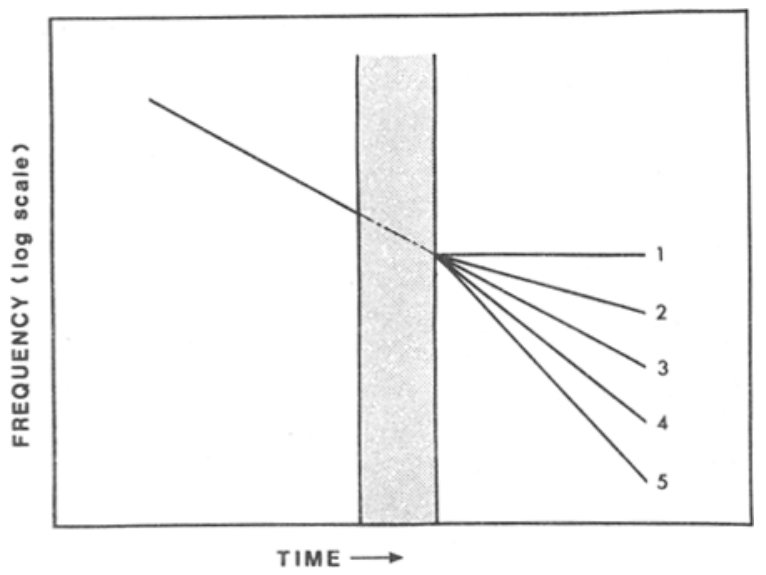

Figure 9. The levels of the slope-of-the-second-sound factor in Experiment 4 are indicated as follows: (1) 0 slope; (2) $1 / 2$ slope; (3) 1 slope; (4) $3 / 2$ slope; (5) 2 slope.

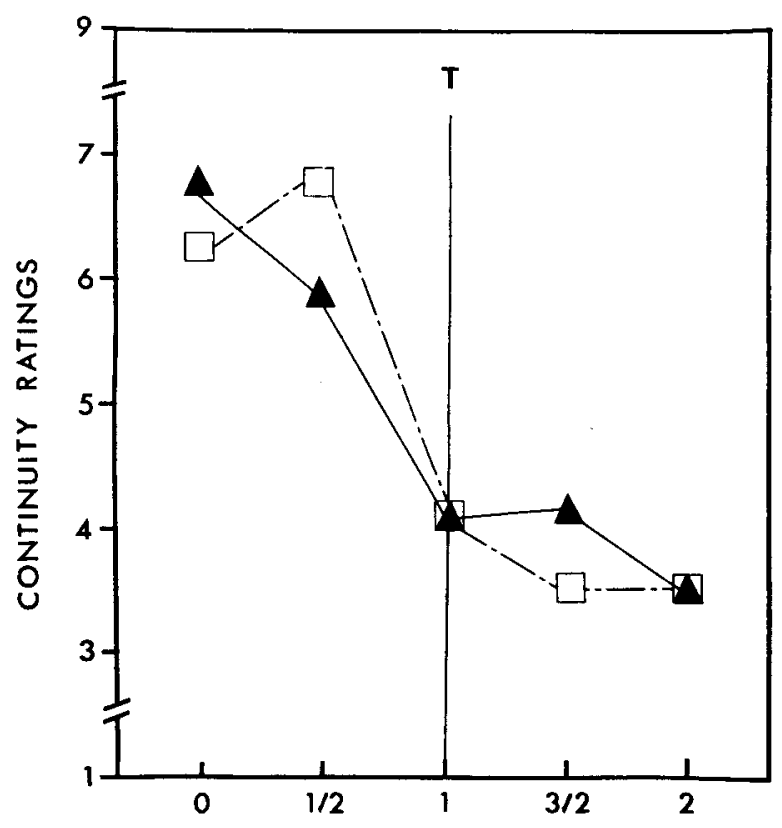

Slope of the post-noise sound

Figure 10. Mean continuity ratings for the conditions in Experiment 4. $\Delta$ : steep prenoise glide; $\square$ : shallow prenoise glide. The vertical line $(T)$ indicates the trajectory conditions of the pastmoise sounds.

540 (trajectory glide), 1086 to 418 , and 1086 to $302 \mathrm{~Hz}$ for the steep glide; 1214,1214 to 1020,1214 to 857 (trajectory glide), 1214 to 720 , and 1214 to $605 \mathrm{~Hz}$ for the shallow glide. The trajectory frequency for the steeper glide was 4.4 semitones lower than its final frequency. The trajectory frequency for the shallow glide was 2.4 semitones below its final frequency. The stimuli were identical in intensity and in spectral characteristics to the ones used in the two preceding experiments. All of the conditions were presented in random order within a session; each condition was presented four times to each subject. Therefore, each subject listened to 40 conditions (10 experimental conditions $\times 4$ repetitions of each condition) in a 13-min session.

Pretest. The training and test procedures were the same as those described in Experiment 3.

Procedure and Apparatus. The procedure and apparatus were identical to those used in Experiment 1.

\section{Results}

The results were analyzed in a two-way ANOVA with the slope-of-the-second-sound factor nested within the slope-of-the-first-glide factor. Mean continuity ratings for each condition are shown in Figure 10. The main effect of the slope of the postnoise glide was highly significant $[F(8,152)=19.39, p<.0001]$. The main effect of slope of the prenoise glide was not significant. Two planned comparisons of conditions of 0 and $1 / 2$ slope versus conditions of $1,3 / 2$, and 2 slope (for each of the two first sounds) resulted in significance at the $\mathbf{. 0 0 0 1}$ level. Pairwise post hoc comparisons among paired conditions of $1,3 / 2$, and 2 slope failed to reach significance at the .05 level. 


\section{Discussion}

The results seem to show that the trajectory-assessment mechanism is not very precisely tuned for slope. Indeed, continuity was equally good for all of the glides having a slope equal to or steeper than that of the first glide. As the slope of the postnoise glide became steeper than that of the trajectory glide, we did not measure any decrease in continuity, for both steep and shallow prenoise sounds (see Figure 10). This could be explained by hypothesizing that steeper postnoise glides are less finely tuned and, consequently, that they accept prenoise glides more easily, in a retrograde fashion. This steepness effect was found to operate in the forward direction in Experiment 3, and it is probably symmetrical in a temporal sense.

On the basis of the results of Experiments 3 and 4, we may conclude that a trajectory-based mechanism determines the areas of acceptance for continuity, based on the slope and on the final and initial frequencies of pre- and postnoise sounds, respectively. Steeper glides, before or after the noise, seem to generate larger frequency regions of acceptance than do shallow glides.

\section{GENERAL DISCUSSION}

The results of the experiments performed in this study show that perceived continuity of the softer sounds through the noise was governed by two competing principles of organization: frequency proximity and the good continuation of a trajectory. A frequency-proximity principle is more effective when the sounds before and after the noise are of different types. On the other hand, a trajectory-continuation principle operates when pre- and postnoise sounds are of the same type (i.e., gliding in the same direction). We provided some evidence showing that the auditory system is capable, under appropriate conditions, of extrapolating trajectories in the frequency domain for the purposes of accepting a second glide as a good continuation of the first. We are making no claims about whether the perceptually restored portion of the glide can be based on the extrapolation of a trajectory. In all the cases we studied, the actual restoration itself could just as easily have been done along a trajectory supplied by "rubber-band" interpolation. The trajectorybased mechanism is more finely tuned for the starting frequency than for the slope of the postnoise glide. The results of our study do not show any effect analogous to the visual Poggendorff illusion.

It is reasonable to assume that a two-stage process determines perceived continuity with our stimuli. At the first stage, the direction of the sounds is detected. Two alternative possibilities are available then: the two sounds have the same, or a different, direction. Psychophysiological studies have collected evidence concerning the existence of direction-sensitive channels in the auditory system (Gardner \& Wilson, 1979); moreover, corresponding results have been obtained with psychophysical methods (Pollack, 1968). At the second stage of processing, if the same alternative is true, continuity is determined by a mechanism that operates on the basis of both the trajectory and the steepness of the pre- and postnoise sounds. On the other hand, if the different alternative is true, a frequency-proximity measurement is given more importance in the perceptual integration of the pre- and postnoise sounds into a single sound.

On the basis of our results, it seems more likely that continuity-generating processes need to analyze the last element of our auditory patterns (i.e., the postnoise sounds) before deciding whether or not a sound continued through the previously presented noise burst. This supports the idea that auditory continuity is determined, in a retrograde fashion, by the last elements of a pattern (Vicario, 1973). Similarly, Warren (1983) suggested that listeners make use of prior or subsequent contextual information for the restoration of the obliterated sounds.

\section{REFERENCES}

Brady, P. T., House, A. S., \& Stevens, K. N. (1961). Perception of sounds characterized by a rapidly changing resonant frequency. Journal of the Acoustical Society of America, 33, 1357-1362.

Bregman, A. S., \& DANnENBring, G. L. (1977). Auditory continuity and amplitude edges. Canadian Journal of Psychology, 31, 151-159.

DANNenbring, G. L. (1976). Perceived auditory continuity with alternately rising and falling frequency transitions. Canadian Journal of Psychology, 30, 99-114.

GARDNER, R. B., \& WILSON, J. P. (1979). Evidence for direction-specific channels in the processing of frequency modulation. Journal of the Acoustical Society of America, 66, 704-709.

HENKE, W. L. (1975). MITSYN: An interactive dialogue language for time signal processing. Cambridge, MA: Massachusetts Institute of Technology, Research Laboratory of Electronics.

Houtgast, T. (1972). Psychophysical evidence for lateral inhibition in hearing. Journal of the Acoustical Society of America, 51, 1885-1894.

Pollack, I. (1968). Detection of rate of change of auditory frequency. Journal of Experimental Psychology, 77, 535-541.

Thurlow, W. R., \& ElFner, L. F. (1959). Continuity effects with alternately sounding tones. Journal of the Acoustical Society of America, 31, 1337-1339.

Vicario, G. (1960). L'effetto tunnel acustico. Rivista di Psicologia, 54, 41-52.

VICARIo, G. (1973). Tempo psicologico ed eventi. Florence: GiuntiBarbera.

WARREN, R. M. (1970). Perceptual restoration of missing speech sounds. Science, 167, 392-393.

WARREN, R. M. (1983). Auditory illusions and their relation to mechanisms normally enhancing accuracy of perception. Journal of the Audio Engineering Society, 31(9), 623-629.

WARREN, R. M., \& OBUSEK, C. J. (1971). Speech perception and phonemic restorations. Perception \& Psychophysics, 9, 358-362.

WARrEN, R. M., ObUSEK, C. J., \& ACKrofF, J. M. (1972). Auditory induction: Perceptual synthesis of absent sounds. Science, 176, 1149-1151.

Weintraub, D., \& Krantz, D. (1971). The Poggendorff illusion: Amputations, rotations and other perturbations. Perception \& Psychophysics, 10, 257-264.

WeRTHEIMER, M. (1912). Experimentelle Studien über das Sehen von Bewegung. Zeitschrift fur Psychologie, 61, 161-265.

(Manuscript received June 9, 1986; revision accepted for publication March 31, 1987.) 